\title{
Lapupilometría en el socorrismo acuático profesional: la vigilancia como factor determinante del ahogamiento \\ The use of pupillometry in aquatic lifesaving: vigilance as a key factor in drowning
}

\author{
*Sergio López García, *María Inmaculada Martín Villar, **José Antonio Navia Manzano, *José *Enrique Moral García, Mario \\ Amatria Jiménez, Rubén Maneiro Dios, ***CristianAbelairas Gómez \\ *Universidad Pontificia de Salamanca (España), **Universidad Politécnica de Madrid (España), ***Universidad de Santiago de Compostela
} (España)

Resumen. Una de las causas de muerte significativa en el mundo actual es el ahogamiento por sumersión, la medida preventiva más eficaz es la figura del socorrista acuático, destacando tres funciones principales: prevención, vigilancia e intervención. Un aspecto importante de la vigilancia es el escaneo, siendo escasos los estudios científicos en el método de ejecutarlo. Centrándonos en la disposición psicológica de vigilar, abarca dos acciones entrelazadas: percibir y responder. La percepción es indispensable para la acción, entre ambas está la toma de decisión. Percibiendo, el ojo realiza movimientos oculares habiendo vinculación entre ellos y el proceso de toma de decisión pasando a ser objeto observable y medible con la pupilometría. De este modo, por medio de la pupilometría podrían descubrirse los aspectos que entraña la vigilancia con el objetivo de prevenir de forma más eficaz el ahogamiento. Por tanto, el objetivo del presente trabajo ha sido organizar y analizar la literatura científica en términos de vigilancia y percepción en socorrismo acuático.

Palabras clave: Ahogamiento, socorrismo acuático, vigilancia, toma de decisión y pupilometría.

Abstract. One of the most important causes of death nowadays is drowning by submersion. Aquatic lifeguards are considered the most effective preventive measure. Lifeguards are entrusted with three main functions: prevention, surveillance, and intervention. One of the most important aspects of surveillance is scanning. However, there are few studies that focus on how to perform it. The psychological aptitude to watch involves two interlocked actions: perceiving and responding. Perception is essential for action, but between both is decision-making. In the action of perceiving, eyes perform eye movements which have a link to the cognitive process of decision making. Eye movements became observable and measurable with the birth of pupilometry. In this way, pupilomety might help to study the links involved in surveillance in order to prevent drowning more effectively. Thus, the aim of this study was to organize and analyze scientific literature in terms of surveillance and perception in lifesaving.

Key words: Drowning, aquatic lifeguard, surveillance, decision making and pupilometry.

\section{Introducción}

Los accidentes son un importante problema de salud a nivel mundial por las elevadas cifras de mortalidad, morbilidad, sufrimiento humano y pérdidas económicas que ocasionan (CDCP, 2004; Morrongiello \& House, 2004), tratándose de una entidad prevenible que si se estudia a profundidad podría intervenirse efectivamente (Torres, Fonseca, Díaz, Del Campo, \& Roché, 2010). Son declarados los accidentes por la Organización Mundial de la Salud(OMS) como acontecimiento fortuito generalmente desgraciado o dañino independiente de la voluntad humana, provocado por una fuerza exterior que actúa rápidamente y que se manifiesta por la aparición de lesiones orgánicas o trastornos mentales (UNICEF, 2001; Hurtado-Sierra, Medina-Chicué, SarmientoLimas \&, Godoy, 2015)

Se acentúa entre los accidentes el ahogamiento por sumersión. Un elevado número de estudios demuestran que es una de las grandes causas de muerte en todo el planeta (Australia, 2004; Peden, \& McGee, 2003; Chalmers, \& Morrison, 2003; Browne, Lewia-Michl, \& Stark, 2003a, 2003b; Lunetta Pentilä, \& Sajantila, 2002; Edmond, Attia, D’Este \& Condon, 2001; Bose, George, \& Joseph, 2000;Lindholm \& Steensberg, 2000; Mackie, 1999; Lunetta, Penttilä, \& Sarna, 1998; Logan, Branche, Sacks, Ryan, \& Peddicord, 1998).

Según la Real Federación Española de Salvamento y Socorrismo y el Consejo Superior de Deportes (2017), un total de 481 personas perdieron la vida en 2017 por causa del ahogamiento. Sin embargo, hay que tomar estos datos con cautela, ya que es una cifra recogida por medio de noticias de prensa, por lo que los datos podrían estar infraestimados. Esto mismo sucede con la estadística de la Asociación Española de Técnicos en Socorrismo Acuático y Escuela Segoviana de Socorrismo (2017), en la que se registraron un total de 544 fallecimientos. . Tomando como referencia al Instituto Nacional de Estadística, 2.732 personas fallecieron en el año 2016 por ahogamiento, sumersión y sofocación accidentales (INE, 2017). En el plano Europeo son entre

Fecha recepción: 16-08-18. Fecha de aceptación: 08-01-19 Sergio López García

sosalvamento@gmail.com
35.000 a 40.000 las personas que pierden la vida ahogadas cada año (ILSE, 2005) alegándose que en todo el mundo hay aproximadamente cuarenta y dos muertes por ahogamiento cada hora, todos los días (WHO, 2014; Barcala-Furelos, et al. 2016). Desde la OMS describen este fenómeno como una importante cuestión pública que reclama atención mundial (WHO, 2008; WHO, 2016), siendo una de las causas de muerte inducida globalizada (WHO, 2017).

Es definido el término de ahogamiento por sumersión como el proceso de experimentar insuficiencia respiratoria por la sumersión o inmersión en un medio líquido, generalmente agua (Van Dorp, Knape, \& Bierens, 2003; Idris, et al., 2003; Beeck, Branche, Szpilman, Modell, $\&$ Bierens, 2005). Se alega que el mayor riesgo de ahogamiento lo presentan los niños, los varones y las personas que tengan fácil acceso al agua (WHO 2011; Vargas, 2015), destacando que los hombres tienen el doble de probabilidad de ahogarse frente a las mujeres (Howland, Hingson, Mangione, Bell \& Bak, 1996; Halik, Poznaríska, Seroka, \& Woityniak, 2014). Los factores que contribuyen en el ahogamiento son la desatención a niños en lugares con agua, abuso de drogas o alcohol, poca habilidad para nadar, agotamiento dentro del agua, traumas, conductas peligrosas, actividades violentas, sumersión voluntaria prolongada e intento de suicidio (Valverde, \& Cordero, 2009; WHO, 2017), acentuándose estos riesgos en las playas turísticas, en donde los bañistas están más expuestos a los mismos (Bordehore,Alonso, SánchezFernández, Canepa, Acevedo, Nogué, \& Fuentes, 2016).

Se considera que el $80 \%$ de todos los ahogamientos se pueden prevenir (Semple-Hess, \& Campwala, 2014), existiendo una figura profesional capaz de llevar a cabo estrategias tanto preventivas como de intervención: el socorrista acuático (Vázquez-González, AbelairasGómez, López-Gacía, \& Palacios-Aguilar, 2017). El socorrista acuático es definido por la Real Academia Española como persona especialmente adiestrada para prestar socorro en caso de accidente (RAE, 2014). Su responsabilidad se centra en vigilar y cuidar las zonas de baño así como en resolver cualquier incidente acuático (Palacios-Aguilar, BarcalaFurelos, López-García, Carpentier \& Abelairas-Gómez, 2018). Su intervención se concreta en una serie de eslabones denominados «cadena de supervivencia del ahogamiento» única y universal, que guían los pasos importantes para salvar vidas; (Szpilman, Bierens, Handley, \& Orlowski, 2012; Szpilman, et al. 2014), siendo imprescindible reducir el 
tiempo de todos los eslabones de la cadena de salvamento para lograr la atención médica temprana (Sanz-Arribas, Aguado-Gómez \& Martínez de Haro, 2017).

No todos los espacios acuáticos son vigilados, y los que se vigilan no están cubiertos las 24 horas (Barcala-Furelos, Abelairas-Gómez, Domínguez-Vila, Vales-Porto, López-García \& Palacios-Aguilar, 2017). La vigilancia de las piscinas se suele realizar en función de lo que indique la normativa a la que esté sujeta la instalación, en el caso de España, depende de la norma específica de cada Comunidad autónoma (Arribas, 2011). Sin embargo, en los espacios naturales no existe una normativa, ni de carácter estatal ni autonómico, llevándose municipalmente bajo una partida presupuestaria (López,Abelairas, Moral, Barcala \& Palacios, 2016).

El servicio de salvamento y socorrismo influyen positivamente en el descenso de accidentes por ahogamientos (Abraldes, 2006; Palacios \& Barcala, 2008), puesto que realiza una función importante de prevención, vigilancia e intervención en el caso de ocurrir (Abraldes, Manzanares \& Fernández, 2009). Pormenorizando en estas tres funciones, hoy por hoy prevención y vigilancia son considerados elementos fundamentales en el salvamento acuático, y sin duda esta es una relación con una fuerte base de sustentación. Sin embargo, es necesario tener en cuenta que la vigilancia precede el acto de prevenir (Fernández-Cano, 2004).

Por otro lado, la intervención de un socorrista cuando detecta un ahogamiento es el rescate acuático, descrito como operación para recuperar a las personas en peligro y cubrir sus necesidades médicas iniciales o de otro tipo (Coastguard US, 2007). Dicha intervención es secuenciada en cinco fases: percepción del problema, análisis de la situación, toma de decisiones, ejecución de las acciones elegidas y evaluación final de la intervención (Palacios, 2011; Palacios \& Barcala, 2008). Estas tres funciones del socorrista acuático: prevención, vigilancia e intervención, tienen como factor común el componente visual, puesto que sin éste no hay posibilidad de ninguna de las tres. De esta forma, la percepción visual se convierte en un elemento imprescindible y destacado para esta profesión.

Los aspectos clave en los que se centrará esta revisión de la literatura serán la vigilancia y la percepción. El objetivo del presente trabajo ha sido organizar y analizar la literatura científica en términos de vigilancia y percepción en el socorrismo acuático para así continuar con la mejora en la eficiencia de esta función.

\section{La vigilancia: estrategias de escaneo y percepción}

Puntualizando en el eje fundamental sobre el que gira la seguridad en el mundo del salvamento acuático, la vigilancia (Fernández-Cano, 2004), es definida como la capacidad de ver y reconocer un evento singular para posteriormente tomar la acción adecuada (NASCO, 2015). Para dicho proceso es necesario la observación, el registro y la evaluación del agua que se está inspeccionando (Page, \& Griffiths, 2014). Dentro de la vigilancia, el aspecto más importante es el escaneo (NASCO, 2015) considerado como el uso del sistema visual para alimentar al cerebro con información sobre el mundo exterior. Este escaneo permite la planificación estratégica y funciones de gestión para el socorrista, dando como resultado un entorno más seguro para los usuarios (Fenner, Leahy, Buhk, \& Dawes, 1999). El escaneo visual requiere que el socorrista realice un barrido a su área de responsabilidad, observando a cada persona o grupo de personas detectando quién necesita ayuda y quién no (International Life saving Federation, \& International Medical Rescue Conference, 1997). Por tanto, la tarea del socorrista abarca un componente de escaneo, de fijación y de evaluación. Las investigaciones parecen indicar que el escaneo realizado por los socorristas es continuo, por lo que los ahogamientos fallidos pueden deberse a una evaluación defectuosa (Lanagan-Leitzel, \& Moore, 2008). Aunque bien es cierto que muchas condiciones pueden afectar a la vigilancia y a la efectividad de un escaneo: la falta de habilidad en cómo reconocer o identificar un ahogamiento, la fatiga fisiológica o psicológica por parte del socorrista, la turbidez del agua, o los factores ambientales (Hunsucker, \& Davison,
2013). Con dichas condiciones, es considerada como una tarea visual desafiante, especialmente cuando el objetivo a detectar entraña un comportamiento inusual, como es el caso del ahogamiento (Schwebel, Lindsay, \& Simpson, 2007).

Adentrándonos específicamente en la planificación estratégica del escaneo en socorristas acuáticos, en las últimas décadas se han propuesto numéricamente cuatro modelos para la realización de este proceso de vigilancia (Griffiths, 2002).

Inicialmente, en la década de los 70, las primeras contribuciones significativas fueron realizadas por Frank Pia, quién introdujo el término de «respuesta instintiva de ahogamiento» (RIA) describiendo el patrón y las características de un persona en proceso de ahogamiento (Pia, 1974). Posteriormente desarrolló el modelo RID para ayudar a explicar las razones por las que puede ocurrir el ahogamiento incluso cuando el socorrista está presente. Sugirió que el ahogamiento puede ocurrir por falta de reconocimiento de los síntomas de ahogamiento, intrusión y distracción dentro de la labor de vigilancia (Avramidis, Butterly, \& Llewellyn, 2007; Lifesaving society, 2013). Un resultado importante de sus trabajos fue conocer el corto periodo de tiempo en el que una víctima puede permanecer en la superficie y por lo tanto la dificultad de percibirla (Griffiths, 2002), siendo numerosos escritos los que reafirman que la víctima consciente sólo puede luchar en la superficie del agua durante 20-60 segundos (Fenner, Leahy, Buhk, \& Dawes, 1999; Rodríguez-García, \& Gómez-García, 2014).

Posteriormente tomando como referencia el escaso tiempo en el que se produce el proceso de ahogamiento, Ellis y White introdujeron la llamada «Regla de Protección 10/20»que recomendaba un límite de tiempo de 10 segundos para escanear e identificar a una persona en peligro de ahogamiento y un límite de 20 segundos para acercarse y tomar contacto con la víctima. (Ellis, \& White, 1994). Más tarde, Griffiths desarrollóla «Estrategia de escaneo de cinco minutos» (Fagan, \& Griffiths, 2003; Griffitths, \& Griffiths, 2013) desarrollada para ayudar a centrarse en los patrones de los bañistas en el agua, de acuerdo a esta estrategia los socorristas deberían de cambiar su posición, el escaneo y postura cada cinco minutos para mantenerse vigilantes y efectivos (Griffiths, 2000; Connolly, 2012).

Recientemente, se ha desarrollado el modelo « $4 W »$, con el objetivo de ofrecer una forma alternativa para comprender los antecedentes del proceso ahogamiento y ayudar al campo de la seguridad acuática (Avramidis, 2009). En este método se estudian cuatro factores como principales determinantes del accidente acuático: el socorrista, la víctima, el lugar y las circunstancias (Avramidis, Buttterly \& Llewellyn, 2009a; 2009b, 2009c, 2009d; Avramidis, et al., 2010).

Dichos modelos mencionados anteriormente, desde los setenta hasta la actualidad, están limitados debido a que en su mayoría no han sido confirmados empíricamente y su efectividad ha sido cuestionada (DeRosa, 2008; Ellis and Associates, 2001). Es decir, dichas teorías sobre los procedimientos de escaneo han pasado la prueba del tiempo, aunque sin embargo existe una sorprendente escasez de estudios sobre los métodos de vigilancia. Por tanto, parece necesario que se dedique una mayor atención a este tema (Brewster, 1999).

\section{Percepción visual y movimientos oculares}

El análisis de la vigilancia requiere prestar atención a los movimientos oculares, cuya investigación ha evolucionado enormementeen losúltimos años (Parra, 2004). La gran importancia del sistema visual dentro del proceso perceptivo ha llevado a los investigadores a analizar los movimientos oculares de los seres humanos en diversos entornos, proporcionando una fuerte evidencia acerca de la atención visual y la conducta visual (Barber \& Legge, 1980). Por ello, en la literatura científica se encuentran diferentes experiencias sobre el seguimiento visual, la sincronía entre respuesta y objetivo en movimiento, la toma de decisiones, la anticipación visual y la relación entre el movimiento cabezaojo(Kelling \& Corso, 2018). La significación de los movimientos oculares para evaluar la actividad humana reside en que representan una cantidad manifiesta, observabley, por tanto, medible asociada con las interacciones, 
y la relación predecible con los procesos cognitivos asociados con la atención visual (Gentil, 2017). También se destaca conocer la fijación de la mirada, puesto que lo que está siendo fijado por los ojos indica aquello que está siendo procesado por la mente (Scheiter \& Van Gog, 2009). Por ejemplo, en el mundo del deporte, la pupilometría se ha utilizado de forma prolífica en diversas investigaciones mediante el estudio de las fijaciones oculares y sus características contextualizadas en los procesos cognitivos de percepción, toma de decisión y acción motora (Vickers, 2007; Vickers, 2016; Castro, Costa, Lage, Praça, Fernández-Echevarría, Moreno, \& Greco, 2017).

En cuanto al socorrismo acuático, se afirma que las técnicas de escaneo son los movimientos oculares utilizados durante este proceso (Fenner, et al., 1999). Por tanto, dichos movimientos oculares pasan a ser variables primordiales a estudiar en el proceso de vigilancia. Centrándonos en la tipología de los movimientos oculares, se destacan principalmente tres tipos: (i) las fijaciones, que se caracterizan por el mantenimiento del ojo sobre un punto en la escena, (ii) los movimientos sacádicos, definidos como desplazamientos rápidos de los ojos entre dos puntos de fijación, y (iii) los movimientos de seguimiento que se producen cuando el ojo sigue un objeto del entorno que se mueve a una velocidad moderada(Gila, Villanueva \& Cabeza, 2009). En las fijaciones oculares los ojos permanecen relativamente estables para enfocar una zona concreta de la escena durante periodos de tiempo muy breves. Así pues el término «fijación» no denota que el ojo este totalmente estacionario. Durante lo que denominamos fijaciones oculares se ocasionan micromovimientos de fijación involuntarios, con una amplitud generalmente inferior a $1^{\circ}$ de ángulo visual, y cuya misión es ubicar la imagen los más exactamente posible en la fóvea (Cabestrero, CondeGuzón, Crespo, Grzib \& Quirós, 2013).

Las fijaciones visuales que establecen el área de objetivo en la fóvea proporcionan información detallada del área visualizada (Ávila, \& Moreno, 2003). La localización de las fijaciones visuales generalmente se considera como un índice de la importancia relativa de una señal dada dentro de una visualización de estímulo, mientras que el número y la duración de las fijaciones registradas reflejan las demandas de procesamiento de información en el perceptor (Moreno, Saavedra, Sabido, Luis, \& Reina, 2006). Estudios acerca del movimiento ocular en el deporte (Bard \& Carriere, 1975; Bard \& Fleury, 1981; Helsen \& Pauwels, 1993; Williams, Davids, Burwitz, \& Williams, 1994) se han centrado en el análisis de estrategias visuales en situaciones específicas de resolución de problemas. En algunas situaciones de precisión (e.g. tiro libre de baloncesto), se ha encontrado que el patrón visual más efectivo se caracteriza por pocas fijaciones de gran duración (Vickers, 2007). Sin embargo, en situaciones de observaciones de escena con un gran número de estímulos, como es el caso de la vigilancia en socorrismo, no parece que exista un consenso sobre qué estrategia visual es la mejor (Williams, Williams, \& Pascoe, 1999). Por tanto, permanece abierta la línea de investigación relativa a las características de las fijaciones oculares durante una vigilancia realizada por un socorrista. De este modo, se puede llegar a originar una estrategia visual científica que mejore una toma de decisión para una rápida e eficiente respuesta en un corto periodo ante la visualización de la situación de un ahogamiento.

\section{Percepción, atención y toma de decisión: vigilancia}

Entendiendo la vigilancia como la actividad por la que el socorrista percibe lo que ocurre (Pascual-Gómez, 1997), se alega que numerosos accidentes acontecen no porque el suceso no se haya visto, sino porque no se percibió como peligroso (Griffiths, 2008). Mackworth expuso que la vigilancia es una palabra útil para describir una disposición psicológica de percibiry responder (Mackworth, 1948). En lo referente al socorrismo acuático, las innumerables variables existentes hacen que la capacidad de un socorrista para procesar información, permanecer atento y emitir juicios sólidos se vea comprometida (Page, Bates, Long \& Tipton, 2011).

Centrándonos por tanto, en la primera acción que compone la vigilancia, percibir, es definida la percepción como el proceso psicológico, a través del cual el perceptor interpreta y atribuye un sentido a la información que le llega a través de distintas modalidades sensoriales (vista, oído, tacto, olfato, gusto) (Ballesteros, 2002). Destacando el primer sentido sensorial mencionado, la vista, debido a que se ha convertido en el sentido más relevante puesto que la mayor parte de las actividades que el ser humano realiza dependen de la información que llega a la retina, y que luego es seleccionada y procesada por la corteza visual (Purves, 2007). Cabe mencionar, que para poder ver, el ojo tiene que moverse (Ariza \& Magnolia, 2009) siendo los movimientos de los ojos, un elemento central de la visión, no sólo por su frecuencia, sino sobre todo porque su ausencia impide la visión (Pritchard, 1972). Los movimientos oculares se han considerado como un complemento de la percepción visual que busca información (Goodale, 2011).

La percepción visual se basa en el conocimiento del mundo físico que nos rodea a partir de la disposición óptica, es decir, del complejo patrón de la luz reflejada por los diferentes elementos que lo componen obteniendo información sobre el entorno (De la Torre, 2015). Por lo tanto, la percepción visual es la encargada de reconocer, discriminar, e interpretar los estímulos visuales asociándolos a experiencias vividas (Bravo, 2004). En ese proceso de discriminación de los estímulos, el proceso cognitivo que se realiza es la atención visual, siendo explicada como «el proceso cognitivo que facilita la detección de estímulos en una escena visual compleja, como la que habitualmente nos presenta el medio externo» (Ling, \& Carrasco, 2006). Ampliando dicho concepto de atención visual, es considerada como un mecanismo central de control del procesamiento de información, que actúa de acuerdo con los objetivos del organismo activando e inhibiendo procesos, y que puede orientarse hacia los sentidos, las estructuras de conocimiento en memoria y los sistemas de respuesta (Colmenero, Catena, \& Fuentes, 2001).

Centrándonos en una segunda instancia en la última acción que constituye la vigilancia, responder, diversas teorías del procesamiento visual (Shadlen, \& Newsome, 1996; Romo, \& Salinas, 1999; Schall, \& Thompson, 1999) sugieren una distinción entre dos mecanismos diferentes: un proceso perceptivo que extrae información, explicado anteriormente, seguido por un proceso de decisión del alto nivel que evalúa la relevancia de esta información visual, en términos de los objetivos y expectativas, para preparar y generar la respuesta conductual apropiada (VanRullen, \& Thorpe, 2001). Puntualizando en este último mecanismo previo a la respuesta, la toma de decisión, su estudio se ha producido principalmente en tres áreas: la economía, las matemáticas y la psicología, (León, 1987). En cuanto a la psicología, las teorías psicológicas tratan de explicar cómo las personas toman decisiones, en particular en los procesos cognitivos que subyacen en la elección de las mismas (Beresford \& Sloper, 2008). Es declarada la toma de decisión como el acto de elegir entre alternativas posibles sobre las cuales existe incertidumbre (Colmenarez, 2016). Por lo que, tomar una decisión implica que hay opciones alternativas a considerar, y en ese caso, no solo queremos identificar tantas de estas alternativas como sea posible, sino elegir la que mejor se adapte a nuestras metas, objetivos, deseos, valores, y etc. (Fülöp, 2005). Se afirma que dicho proceso consta de varios pasos: Identificación de problemas, generación de alternativas, evaluación de alternativas, elección de una alternativa, implementación de la decisión y evaluación de la efectividad de la decisión (Lunenburg, 2010). En contraposición, la visión de Klein en la toma de decisiones (Klein, 2000), defiende que en algunas situaciones como las de un alto nivel de incertidumbre, no se generan numerosas alternativas, sino que se genera una rápida idea del proceso de actuación ante el problema sin tener en cuenta las posteriores.

En socorrismo, la toma de decisión representa el tercer paso en la secuenciación del rescate acuático y la etapa previa a la ejecución de la respuesta donde se seleccionan las acciones adecuadas siendo señalado como «el momento más importante, porque es el acto en el cual decidimos, cuál será el procedimiento y la forma de reaccionar en un evento de esta magnitud» (Oses \& Quirós, 2004). Cabe destacar que esta profesión desempeña una labor semejante a las fuerzas de seguridad del estado debido a su completa dedicación a la seguridad de las comunidades y acentuando el fin de velar por la seguridad de las personas (United 
States Lifesaving Association, 1981; Abelairas, García, López, \& Rial, 2012).

Relacionando el proceso de toma de decisiones y los equipos de emergencia nos encontramos que las fuerzas armadas estadounidenses optaron por el empleo, desarrollo y evolución de un modelo sobre el proceso que se realiza en la toma de decisiones, más específicamente en el reconocimiento primario en la decisión (RPD) «Lo primero que se nos pasa por la cabeza» (Klein, 1986; Klein, 1993; Klein, 2000). Ampliando la visión de Klein y sus colaboradores, definieron el modelo de decisión dereconocimiento primario como aquel en el que se describen las decisiones llevadas a la actuación en una acción, teniendo en cuenta el conocimiento, la formación y las experiencias de las personas (Klein, Calderwood, \& Clinton-Cirocco, 1989). El objetivo primordial de la creación del modelo RPD es lograr un aumento significativo en el ritmo de funcionamiento (OPTEMPO). Para ello es fundamental recopilar, analizar, integrar y aplicar la información que hemos recibido y que nos ayudará a anticiparnos y contrarrestar las amenazas para poder actuar más eficaz (Ross, Klein, Thunholm, Schmitt \& Baxter, 2004). Esa recopilación y análisis para la toma de decisiones, afirman los expertos, que parte de las experiencias para identificar la situación y el plan de acción(COA) que se ejecutará (Killion, 2000). Así, se considera buenas tomadoras de decisiones a aquellas personas que generan un proceso de actuación eficaz y rápido en su primera decisión ante la situación (Klein, Wolf, Militelio \& Zsambok, 1995). En este sentido, otros investigadores defienden que la primera idea de acción no debe representar necesariamente la opción óptima de entre todas las posibilidades, sino que debe ser simplemente suficientemente buena (Johnson \& Raab, 2003).

Por lo tanto, llevándolo a la especificación de la vigilancia de los socorristas, la percepción visual de un ahogamiento viene determinada en primer lugar por el reconocimiento de los factores que se producen en el accidente como es destacada la actitud o estado de la víctima en el agua, definida por Pia como respuesta instintiva al ahogamiento. En segundo lugar, se realiza la discriminación de los estímulos percibidos con el fin de centrar la atención visual en los elementos o acciones que aportan información de la situación. En el último paso de la percepción entra la interpretación junto al entendimiento con ayuda de la experiencia de que un usuario está sufriendo dicho acontecimiento.

Una vez se han percibido los estímulos que acompañan a un ahogamiento, es decir, la identificación del problema, el socorrista comenzará a formar un sistema de respuesta ante ese evento con ayuda del análisis de la situación, siendo dicha respuesta precedida por el proceso de toma de decisión seleccionado según las circunstancias para elegir las acciones adecuadas. En ese proceso de toma de decisión se crearán diferentes alternativas, seleccionando entre las opciones que se plantean la mejor acción según las circunstancias del momento. En una última instancia se implementará la decisión elegida con la intervención por parte del socorrista realizando las acciones elegidas.

\section{Conclusiones}

El ahogamiento es un problema de preocupación a nivel mundial debido a la ocupación entre los primeros puestos como causa de muerte. En la lucha contra el ahogamiento por sumersión destaca como pieza de prevención y seguridad fundamental, la presencia de los socorristas acuáticos tanto en instalaciones acuáticas como en espacios naturales con la afirmación de que gracias a dicha presencia se ha reducido el número de accidentes acuáticos. En dicha figura a la que nos referimos como es el socorrista acuático, recaen tres funciones primordiales dentro de su labor: vigilancia, prevención eintervención. Estas funciones guardan una correlación estrecha entre ellas, puesto que sin la vigilancia no sería posible la realización de una prevención, de la misma forma que sin una percepción antecesora mediante la vigilancia de un ahogamiento no se podría llevar a cabo una intervención conocida específicamente como rescate acuático, siendo esta la primera fase en la secuencia de actuación en la intervención de un socorrista. Es por tanto destacable dentro de la función de la vigilancia el órgano sensorial, y el proceso partícipe de dicha acción; como es la vista y la percepción puesto que sin ambos elementos no sería posible el reconocimiento junto a la interpretación de los estímulos visuales cuando acontece un ahogamiento.

La percepción inmediata de un proceso de ahogamiento supondrá tener más posibilidades de evitar la muerte del ahogado. Esto cobra todavía más importancia si es sabido que la víctima solamente logrará mantenerse en la superficie de 20 a 60 segundos. El término específico asignado que hace referencia a la forma o manera para realizar la vigilancia es el escaneo siendo sinónimo de estrategia o planificación visual para realizar la acción de vigilar de forma eficaz, siendo escasos los modelos de escaneo propuestos desde su inicio en la década de los setenta hasta la actualidad, contablemente cuatro: en primer lugar el modelo RID por Frank Pía, seguidamente la Regla de protección 10/20 por Ellis y White, posteriormente el escaneo de cinco minutos de la mano de Griffiths, y enúltimo lugar el modelollamado 4W.Dichos modelos anteriores guardan características comunes referidas a su limitación, ya que por un lado son escasos los estudios específicos realizados en esta línea de investigación, y por otro lado la efectividad cuestionada de los modelos originados debido a una falta de sustentación científica.

En busca de un mayor aporte informativo sobre lo que conlleva la acción de vigilar encontramos que entraña aparte de percibir y escanear, una disposición psicológica de responder. Esta respuesta aguarda un complejo proceso intrínseco precedente como es la toma de decisión que conlleva elegir entre varias opciones la alternativa más eficaz acorde al objetivo de evitar la pérdida de una vida frente al ahogamiento. Esta situación extrema con gran incertidumbre donde es vital la correcta e imprescindible actuación, nos lleva a detenernos en el proceso de toma de decisión ante situaciones extremas. En este contexto, nos encontramos con la limitación de que son escasos los modelos identificados, siendo únicamente encontrado el modelo de decisión de reconocimiento primario (RPD) descrito por Gary Klein teniendo como objetivo estudiar y comprender el acto en el que se realiza la decisión en situaciones con incertidumbre donde prima el corto periodo de tiempo de reacción eficaz. Siendo de forma concluyente que la mejor toma de decisión es aquella capaz de tener un carácter variable acorde a los estímulos cambiantes en el ambiente.

Ante el papel primordial que presenta el sistema visual junto a la estrecha relación entre la toma de decisión y acción ha llevado a los investigadores a analizar los movimientos oculares dentro delapsicología cognitiva, habiendo una fuerte evidencia de la relación de los procesos cognitivos hablados con dichos movimientos. Los movimientos oculares junto a las fijaciones entre los mismos pasan a ser objetos observables y medibles a partir dela década de los sesenta con el origen delapupilometría. Las características que describen a las fijaciones (localización, número, duración) han sido objeto destacado de estudio en el contexto deportivo, permitiendo analizar las estrategias visuales a la hora de percibir de forma hábil un estímulo y responder con una acción motora de forma eficaz en el tiempo exacto. Llevándolo a la especificación del socorrismo acuático, se podrían analizar las estrategias visuales mediante la pupilometría para evaluar si la vigilancia se realiza de forma eficiente. Destacando que aun siendo la vigilancia el eje fundamental por el que gira la seguridad en el medio acuático, se afirma que rara vez se instruye (Page, \& Griffiths, (2014). A los socorristas se les enseña en torno a esta temática únicamente el conjunto específico de comportamientos que indican un evento crítico y que supone un ahogamiento (Respuesta instintiva al ahogamiento,) llevándose el mayor enfoque y protagonismo en el proceso de capacitación y certificación las técnicas de rescate y los primeros auxilios.

\section{Referencias}

Abelairas, C., García, M., López, S., \& Rial, T. (2012). Metodología de enseñanza en Salvamento Acuático profesional. Trances 4(4) 285302. Recuperado de: http://www.trances.es/papers/ TCS\%2004_4_2.pdf

Abraldes, J.A. (2006). Salvamento y socorrismo. Secuencias de rescate en el medio acuático. (1 ed.) La Coruña: Federación de Salvamento 
y Socorrismo de Galicia.

Abraldes, J.A., Manzanares, A., \& Fernández, F. (2009). Análisis de los rescates en un parque acuático. Ciencia, Cultura y Deporte 4(12) ,173-178. Recuperado de: http://www.redalyc.org/service/ redalyc/downloadPdf/1630/163013073006/1.

Asociación Española de Técnicos en Socorrismo Acuático y Escuela Segoviana de Socorrismo (2017). Datos y análisis de Ahogamientos del año 2017. Recuperado de: https://drive.google.com/file/d/ 1_WUk9fAUDARol4Iel6LGzfSQJjYeqGjG/view

Ariza,A., \& Magnolia, S. (2009). Introducción a la Ingeniería Biomédica. Ingeniería electrónica. Universidad Pontificia Bolivariana, Bucaramanga.

Arribas, I. (2011). La coordinación de socorristas en piscinas con grandes láminas de agua. Revista Internacional de Medicina y Ciencias de la Actividad Física y el Deporte 11(44), 650-673.

Recuperado de:Http://cdeporte.rediris.es/revista/revista44/ artcoordinacion198.htm

Australia, T.R.I.S.S. (2004). The National Drowning Report 2004.

Ávila,F., \& Moreno, F.J. (2003). Visual search strategies elaborated by tennis coaches during execution error detection processes. Journal of Human Movement Studies 44, 209-224. Recuperado de: http:// www.ugr.es/ swimsci/SwimmingScience/page4/page60/files/ 2006Moreno_Saavedra.pdf

Avramidis, S., Butterly, R., \& Llewellyn, D. (2007). The 4W Model of drowning. International Journal of Aquatic Research and Education 1(3), 221-230. https://doi.org/10.25035/ijare.01.03.05

Avramidis, S. (2009). The 4W Model of Drowning for Lifesaving of non-Aquatic and Swimming Activities. PhD thesis. Leeds Metropolitan University.

Avramidis, S., Butterly, R., \& Llewellyn, D. (2009a). Drowning incident rescuer characteristics: Encoding the first component of the $4 \mathrm{~W}$ model. International Journal of Aquatic Research and Education 3(1), 66-82. https://doi.org/10.25035/ijare.03.01.06

Avramidis, S., Butterly, R., \& Llewellyn, D. (2009b). Who drowns? Encoding the second component of the 4W model. International Journal of Aquatic Research and Education 3(3), 224-235. https:/ /doi.org/10.25035/ijare.03.03.03

Avramidis, S., Butterly, R., \& Llewellyn, D. (2009c). Where do people drown? Encoding the third component of the $4 \mathrm{~W}$ model. International Journal of Aquatic Research and Education 3(3), 236-254. https://doi.org/10.25035/ijare.03.03.04

Avramidis, S., Butterly, R., \& Llewellyn, D. (2009d). Under what circumstances do people drown? Encoding the fourth component of the 4W model. International Journal of Aquatic Research and Education 3(4), 406-421. https://doi.org/10.25035/ijare.03.04.08

Avramidis, S., et al. (2010). Crucial findings from the 4W model of drowning for practical and teaching applications. En Kjendlie, R.K., et al. (Eds.) Proceedings of the 40th International Symposium for Biomechanics and Medicine in swimming 2010. Oslo, Norwegian School of sport Sciences, 354-356.

Ballesteros, S. (2002). Psicología General: Atención y percepción. Vol:II. UNED.

Barber, P.J., \& Legge, D.(1980). Percepción e información. México: Compañía Editorial Continental, S.A.

Barcala-Furelos, R., Szpilman, D., Palacios-Aguilar, J., Costas-Vegas, J., Abelairas-Gomez, C., Bores-Cerezal, A., ...\& Rodríguez-Nuñez, A. (2016). Assessing the efficacy of rescue equipment in lifeguard resuscitation efforts for drowning. Am J Emerg Med 34(3), 480485.

Barcala-Furelos, R., Abelairas-Gómez, C., Domínguez-Vila, P., ValesPorto, C., López-García, S., \& Palacios-Aguilar, J. (2017). Policía costera de Vigo. Estudio piloto cuasi-experimental sobre rescate y RCP. Revista Internacional de Medicina y Ciencias de la Actividad Física y del Deporte 17(66), 379-395. Recuperado de: Http:// cdeporte.rediris.es/revista/revista66/artpolicia800.htm

Bard, C. \& Carriere, L. (1975). Etude de la prospection visuelle dans des situations problemes en sports. Mouvement, 10, 1523-1537.

Bard, C. \& Fleury, M. (1981). Considering eye movement as a predictor of attainment. En I. M. Cockerill, y W. W. MacGillivary (Eds.),
Vision and Sport, 28-41. Chentelham (UK): Stanley Thormes.

Beeck, E.F., Branche, C.M., Szpilman, D., Modell, J.H., \& Bierens, J.J.L.M. (2005). A new definition of drowning: Towards documentation and prevention of a global public health problems. Bulletin World Health Organization 83(11), 853-856.

Beresford, B., \& Sloper, T. (2008). Understanding the Dynamics of decision-Making and Choice:Ascoping Study of Key Psychological Theories to Inform the Design and Analysis of the Panel Study. Social Policy Research Unit, University of New of York.

Bordehore, C.,Alonso, C., Sánchez-Fernández, L., Canepa,A., Acevedo, M., Nogué, S., \& Fuentes, V. L. (2016). Lifeguard assistance at Spanish Mediterranean beaches: Jellyfish prevail and proposals for improving risk management. Ocean \& Costal and Management $131,45-52$

https://doi.org/10.1016/j.ocecoaman.2016.08.008

Bose, A., George, K., \& Joseph, A. (2000). Drowning in childhood: a population based study. Indian Pediatric 37(1), 80-83.

Bravo, L. (2004). Las destrezas perceptuales y los retos en el aprendizaje de la lectura y escritura. Una guía para la exploración y comprensión de dificultades específicas. Actualidades Investigativas en Educación 4(1), 2-23.

Brewester, B.C. (1999). Prevention of drowning: Visual scanning and attention span in lifeguards. Journal of Occupational health and safety.Australia.

Browne, M.I., Lewis-Michl, E.I., \& Starks, A.D. (2003a). Unitentional drowning among New York state residents, 1988-1994. Public health Reports 118(5), 448-458. https://doi.org/10.1016/S00333549(04)50276-5

Browne, M.I., Lewis-Michl, E.I., \& Starks, A.D. (2003b). Watercaft related drowning among New York state residents, 1988-1994. Public Health Reports 118(5), 459-463. https://doi.org/10.1016/ S0033-3549(04)50277-7

Cabestrero, R., Conde-Guzón, P.A., Crespo, A., Grzib, G., \& Quirós, P. (2013). Fundamentos psicológicos de la Actividad Cardiovascular y Oculomotora. Madrid, España:UNED.

Castro, H. O., Costa, G. C., Lage, G. M., Praça, G. M., FernándezEchevarría, C., Moreno, M.P., \& Greco, P.J. (2017). Comportamiento visual y toma de decisions en situaciones de ataque en voleibol. Revista Internacional de Medicina y Ciencias de la Actividad Física y del Deporte. In Press. http://cdeporte.rediris.es/ revista/inpress/artcomportamiento1040e.pdf

Centers for Disease Control and Prevention (2004). Non fatal and fatal drowings in recreational water settings. United States, 2001-2002. Morbidity and Mortality Weekly Report 53(21), 447-452.

Chalmers, D.J., \& Morrison, I. (2003). Epidemiology of non submersion injuries in aquatic sporting and recreational activities. Sport Medicine 33(10), 745-750. https://doi.org/10.2165/00007256-20033310000003

Coastguard US. (2007). National searchand rescue plan for the United States. US Coastguard, Washington, DC.

Colmenero, J.M., Catena, A., \& Fuentes, L.J. (2001). Atención visual: Una revisión sobre las redes atencionales del cerebro. Anales de psicología 17(1), 45-67. Recuperado de: http://www4.ujaen.es/ jjimenez/redes.pdf

Connolly, J. (2012). The C-Zones Framework. International Journal of Aquatic Research and Education 6(1), 80-93. https://doi.org/ 10.25035/ijare.06.01.09

Colmenarez, C. (2016). La Toma de decisiones grupales. Revista Digital ISSU. Universidad Fermín Toro.

De la Torre, O. (2015). Percepción de los actores del proceso de aprendizaje del área de historia, geografía y economía tercer grado de la institución educativa los chankas de quillabamba. Perú.

DeRosa, S.P. (2008). Demystifying the protection rule 10/20. En Avramidis,S. (ed.). Handbook on safety and lifesaving. Stamoulis Publishing.

Edmond, K.M., Attia, J.R., D’Este, C.A., \& Condon, J.T. (2001). Drowning and near-drowning in Northern territory children. Med $J$ Aust 175(1), 605-608.

Ellis and Associates (2001). First on-site study of vigilance shows 
lifeguards can't possibly see everything all of the time. Bologne (France).

Ellis, J., \& White, J. (1994). National pool and waterpark lifeguard/ CPR training. Boston,MA: Jones and Barlett Publishers.

Fagan, C., \& Griffiths, T. (2003). Five minute scanning strategy.

Fenner, P., Leahy, S., Buhk, A., \& Dawes, P. (1999). Prevention of drowing: visual scanning and attention span in Lifeguards. The Journal of Occupational Health and safety- Aust NZ, 15, 61-66.

Fernández-Cano, J. (2004). 10 Pasos útiles para una vigilancia efectiva. VI Congress of drowning, lifesaving and water rescue. Escuela Segoviana de Socorrismo.

Fülöp J. (2005). Introduction to Decision Making Methods . Laboratory of Operations Research and Decision Systems: Computer and Automation Institute, Hungarian Academy of Sciences 1.

Gentil., R. Ma . (2017). Registro de patrones de lectura con dispositivos de eye trackers d bajo coste y estudio de su aplicación para la recomendación de diagnóstico de patologías. Grupo de Neurocomputación Biológica. Escuela Politécnica Superior. Universidad Autónoma de Madrid.

Gila, L., Villanueva, A., \& Cabeza, R. (2009). Fisiopatología y técnicas de registro de los movimientos oculares. Anales del Sistema Sanitario de Navarra 32(Suppl 3), 9-26. https://doi.org/10.4321/S113766272009000600002

Goodale, M.A. (2011). Transforming vision into action. Vision Research 51(13), 1567-1587. https://doi.org/10.1016/j.visres.2010.07.027

Griffiths, T. (2000). Five-minute scan. Aquatics International 12.

Griffiths, T. (2002). A master Scan. Aquatics International.

Griffiths, T. (2008). Recreation to Hunsucker and Davison's «Vision and signal detection». International Journal of Aquatic Research and education 2(9), 75-77.

Griffiths, R.C., \& Griffiths, T. (2013). Internal Noise Distractions in Lifeguarding. Aquatic Research and Education 7(6), 56-71.

Halik, R., Poznañska, A., Seroka, W., \& Wojtyniak, B. (2014). Accidental drowning in Poland in 2000-2002. Przegl Epidemiol 68(3), 493499. Recuperado de: 0000-0001-6678-0239

Helsen, W. \& Pauwels, J. M. (1993). The relationship between expertise and visual information processing in sport. En J. Starkes, \& F. Allard (Eds.), Cognitive issues in motor expertise 102, 109-134. New York: Nort-Holland. https://doi.org/10.1016/S01664115(08)61468-5

Howland, J., Hingson, R., Mangione, T.W., Bell, N., \& Bak, S. (1996). Why are most drowning victims men? Sex differences in aquatic skills and behaviors. American Journal Public Health 86(1), 93-96. https://doi.org/10.2105/AJPH.86.1.93

Hunsuncker, J., \& Davison, S. (2013). Scan time goals with analysis of scan times from Aquatic facilities. International Journal of Aquatic Research and Education 7(3), 227-237. DOI: 10.25035/ ijare.07.03.06

Hurtado-Sierra, D.E. Medina-Chicué, E.M., Sarmiento-Lima, C.A., \& Godoy, J.A. (2015). Factores de riesgo relacionados con accidentes pediátricos en un hospital infantil de Bogotá. Revista Salud pública 17(1), 74-84. https://doi.org/10.15446/rsap.v17n1.37064

Instituto Nacional de Estadística (2017). Defunciones según la causa de muerte. Año 2016. Nota de prensa. https://www.ine.es/prensa/ edcm_2016.pdf

Idris, A.H., Berg, R.A., Bierens, J., Bossaert, L., Branche, C.M., Gabrielli, A.....\& Modell, J.H. (2003). Recommendations guidelines for uniform reporting of data from drowning: The Utstein style. Resuscitation 59(3), 45-57. https://doi.org/10.1016/ j.resuscitation.2003.09.003

International Life Saving Federation of Europe (2005). Safety on European Beaches: Operational Guidelines. Germany:ILSE.

International Life Saving Federation, \& International medical rescue conference. (1997). Recognition and observation of potential rescue victims in an open water enviroment. San Diego (California).

Johnson, J.G. \& Raab, M. (2003). Take the First: Option Generation and Resulting Choice. Organizational Behavior and Human Decision Processes 13(62), 25-32. https://doi.org/10.1016/S0749-

\section{8(03)00027-X}

Kelling, N.J., \& Corso, G.M. (2018). The effect of spatial working memory capacity on ball flight perception. Journal of Human Sport and Exercise 13(4), 752-765. https://doi.org/10.14198/ jhse.2018.134.04

Killion, T. (2000). Decision making and the levels of War. Military Review, 66-70.

Klein, G (1986). Recognition-primed decisions in Advances in ManMachine Systemas Research. Greenwich, Reino Unido:W.B.Rouse,

Klein, G. (1993). A recognition-primed decision (RPD) Model of rapid decision making. En Klein, et al (eds). Decision Making in Action: Models and Methods. Ablex Publishing, Norwood (Nueva Jersey).

Klein, G. (2000). Sources of power: How people Make Decisions. MIT, Cambridge, MA.

Klein, G., et al. (1989). Rapid Decisionmaking on the Fireground: proceedings, Human Factors and Ergonomics Society. 30th Annual Meeting, Dayton 1, 576-80.

Klein, G, Wolf, S., Militello, L., \& Zsambok, C. (1995). Characteristics of Skilled Option Generation in Chess. Organizational Behavior and Human Decision Processes 13(62), 63-69. https://doi.org/ 10.1006/obhd.1995.1031

Lanagan-Leitzel, L.,\& Moore, C. (2008). Novice and expert performance on a computerized lifeguarding task. Journal of Vision 8(6). https:/ /doi.org/10.1167/8.6.318

León, O. (1987). La toma de decisiones individuales con riesgo desde la psicología. Infanciay Aprendizaje30, 81-94. Universidad Autónoma de Madrid.

Lifesaving Society. (2013). 10-30 ScanningWindow. Information Bulletin.

Lindholm, P., \& Steensberg, J. (2000). Epidemiology of unintentional drowning and near-drowning in Denmark in 1995. Injury Prevention 6(1), 29-31. https://doi.org/10.1136/ip.6.1.29

Ling, S., \& Carrasco, M. (2006). Sustained and transient covert attention enhance the signal via different contrast response functions. Vision Research 46(9), 1210-1220.https://doi.org/10.1016/ j.visres.2005.05.008

Logan, P., Branche, C., Sacks, J., Ryan, G., \& Peddicord, J.(1998). Childhood drownings and fencing of outdoor pools in the United States, 1994. Pediatrics 101 (6), 3-7. https://doi.org/10.1542/ peds.101.6.e3

López, S., Abelairas, C., Moral, J.E., Barcala, R., \& Palacios, J. (2016). La coordinación de socorristas acuáticos profesionales en espacios acuáticos naturales. Revista Internacional de Medicina y Ciencias de la Actividad Físicayel Deporte 16(62), 403-422. http://dx.doi.org/ 10.15366/rimcafd2016.63.001

Lunenburg, F.C. (2010). The decision making process. National Forum of Educational Administration and supervision Journal 27(4).

Lunetta, P., Pentilä, A., \& Sarna, S. (1998). Water accidents, drowning and alcohol in Finland, 1969-1995. International Journal of Epidemiology 27(6), 1038-1043. https://doi.org/10.1093/ije/ 27.6.1038

Lunetta, P., et al. (2002). Drowning in Finland:»external cause» and injury codes. Injury prevention 8(4), 342-355. https://doi.org/ 10.1136/ip.8.4.342

Mackie, I.J. (1999). Patterns of drowning in Australia, 1992-1997. Medicine Journal Australia 11(171), 587-590.

Mackworth, N.H.(1948). The breakdown of vigilance during prolonged visual search. The Quarterly Journal of Experimental Psychology 1(1), 6-21. https://doi.org/10.1080/17470214808416738

Moreno, F.J., Saavedra, J.M., Sabido, R., Luis, V., \& Reina, R. (2006). Visual search strategies of experienced and nonexperienced swimming coaches. Perceptual and Motor skills 103, 861-872. https://doi.org/10.2466/pms.103.3.861-872

Morrongiello, B.A., \& House, K. (2004). Measuring parent attributes and supervision behaviors relevant to child injury risk: examining the usefulness of questionnaire measures. Injury Prevention 10(2), 114-118. https://doi.org/10.1136/ip.2003.003459

Oses, J. Quirós, R. (2004). Técnicas de rescate en kayak de seguridad en aguas rápidas. Instituto nacional de aprendizaje. 
Page, J., Bates, V., Long, G., Dawes, P., \& Tipton, M. (2011). Beach lifeguards: visual, search patterns, detection rates and the influence of experience. Opthalmic Physiol Opt 31, 216-224. https://doi.org/ 10.1111/j.1475-1313.2011.00824.x

Page, J., \& Griffiths, T. (2014). Scanning and Surveillance: Swimming Pools, Beaches, Open Sea. Heidelberg, Berlín: Springer. https:// doi.org/10.1007/978-3-642-04253-9_47

Palacios, J. (2011). Actualizaciones en técnicas de Socorrismo Acuático: control de víctimas conscientes e inconscientes, traslado, extracción. VI Jornadas de Socorrismo basado en la evidencia científica y Soporte Vital Prehospitalario en el medio acuático. II Ciclo Internacional de Conferencias sobre Socorrismo Acuático. A Coruña. Fundación Universidade da Coruña. ISBN: 978-84-694-3169-6.

Palacios-Aguilar, J., Barcala-Furelos, R., López-García, S., Carpentier, M., \& Abelairas-Gómez, C. (2018). Tabla Air Stand-Up Paddle de rescate acuático: ¿Cómo puede ayudar al socorrista? Revista Internacional de Medicina y Ciencias de la Actividad Física y del Deporte 18(69), 185-197. http://dx.doi.org/10.15366/ rimcafd2018.69.012

Palacios-Aguilar, J., \& Barcala-Furelos, R. (2008). Socorrismo Acuático profesional. Formación para la prevención y la intervención ante accidentes en el medio acuático. $2^{\mathrm{a}}$ Ed. Capítulo 9. Ed: Sadega S.L., A Coruña.

Pascual-Gómez, L.M.,(1997). La Vigilancia en el Salvamento Acuático. Escuela Segoviana de Socorrismo. I Jornadas Técnico-profesionales de salvamento Acuático, Segovia.

Parra, M.A. (2004). Método para el estudio de los movimientos oculares. Colombia Médica. Corporación Editora Médica del Valle 35(2), 55-61.

Peden, M.M., \& McGee, K. (2003). The epidemiology of drowning worlwide. Injury Control and safety Promotion 10(4), 195-199. https://doi.org/10.1076/icsp.10.4.195.16772

Pia, F. (1974). Observations on the drowning of non-swimmers. Journal Physical Education 71(6), 164-167.

Pritchard, R.M. (1972). Stabilized images on the Retina. En Held, R., \& Richards, W. (Eds.) Perception: Mechanisms and models, 176182. San Francisco: W.H. Freeman and Company.

Purves, D. (2007). Neurociencia. Bogotá: Panamericana.

Real Academia Española, (2014). Diccionario de la Lengua Castellana, 23th, Ed. Madrid, España: Espasa-Calpe, S.A.

Real Federación Española de Salvamento y socorrismo, y Consejo Superior de Deportes (2017). Informe Nacional de Ahogamientos producidos del 1 de Enero al 31 de Diciembre del 2017. Recogido en: http://www.rfess.es/DOCUMENTOS/Prevenci\%C3\%B3n/ INA2017.pdf

Rodríguez-García, E. \& Gómez-García, A. (2014). Manejo inicial del casi ahogamiento e hipotermia por inmersión. Scielo 8(1). https:// doi.org/10.4321/S1988-348X2014000100003

Romo, R., \& Salinas, E. (1999). Sensing and deciding in the somatosensory system. Current Opinion in Neurobiology 9, 487493. https://doi.org/10.1016/S0959-4388(99)80073-7

Ross, GK., Klein, G., Thunholm, P., Schmitt, J., \& Baxter, H.C.(2004) The Recognition- Primed Decision Model. Military Review, 6-10.

Sanz-Arribas, I., Aguado-Gómez, R., \& Martínez de Haro, V. (2017) Influencia de las atletas sobre el tiempo de ejecucción en los rescates de víctimas con parada cardiorespiratoria. RETOS 31, 133-136. Recuperado de: file:///C:/Users/Mar\%C3\%ADaInmaculada/

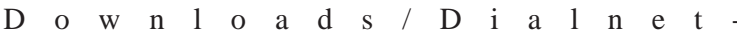
InfluenciaDeLasAletasSobreElTiempoDeEjecucionEnLos5841359\%20(1).pdf

Schall, J., \& Thompson, K. (1999). Neural selection and control of visually guided eye movements. Annual Review of Neuroscience 22(1), 241-259. https://doi.org/10.1146/annurev.neuro.22.1.241

Schwebel, D.C., Lindsay, S., \& Simpson., J. (2007). Brief report: A brief intervention to improve lifeguard surveillance at a public swimming pool. Journal Pediatric Psychology 32(7), 862-868. doi:10.1093/jpepsy/jsm019

Semple-Hess, J., \& Campwala, R. (2014). Pediatric Submersion Injuries:
Emergency care and Resuscitation. EB Medicine 11(6), 1-24. Recuperado de: https://pdfs.semanticscholar.org/80f3/ 19522009713656a440f3b589ae9ec6ced92e.pdf

Shadlen, M., \& Newsome, W.T. (1996). Motion perception: Seeing and deciding. Proceedings of the National Academy of Science U.S.A. 93(2), 628-633. https://doi.org/10.1073/pnas.93.2.628

Scheiter, K., \& Van Gog, T. (2009). Using eye Tracking in applied research to study and stimulate the processing of information from multi-representational sources. Applied Cognitive Psychology 23, 1209-1214. https://doi.org/10.1002/acp.1524

Szpilman D., Bierens, J., Handley, A.J., \& Orlowski, J.P. (2012). Drowning. The New England Journal of Medicine 366(22), 21022110. https://doi.org/10.1056/NEJMra1013317

Szpilman, D., Webber, J., Quan, L., Bierens, J., Morizot-Leite, L., Langendorfer, S.,....\& Løfgren, B.(2014).Creating a drowning chain of survival. Resuscitation 85(9), 1149-1152. https://doi.org/10.1016/ j.resuscitation.2014.05.034

The National Aquatic Safety Company (2015). Our Mission: To Reduce the loss of life due to drowning. NASCO Lifeguard Teextbook.

Torres, M.,Fonseca, C.L., Díaz, M., Del Campo, O., \& Roché, R. (2010). Accidentes en la infancia: Una problemática actual en pediatría. MEDISAN 14(3), 368-378.

UNICEF (2001). A League Table Child Deaths by Injury in Rich Nations. Innocent Report Card.

United States Lifesaving (1981). Lifesaving and Marine Safety. Psicataway, EEUU: New Century Publishers, Inc.

Valverde, C., \& Cordero, T. (2009). Ahogamiento en Pediatría. Revista Medicina Clinica Condes 20(6), 860-867.

Van Dorp, J.C., Knape, J.T., \& Bierens, J.J. (2003). Recommendations: World Congress on Drowning, 2002. The Netherland(Amsterdam)

VanRullen, R., \& Thorpe, S. (2001). The time course of visual processing: From early perception to decision-making. Journal of Cognitive Neuroscience 13(4), 454-461.https://doi.org/10.1162/ 08989290152001880

Vargas, L.A. (2015). La importancia del salvavidas en la gestión de riesgo en medios acuáticos. Sennova, 74-76. https://doi.org/ 10.23850/2422068X.394

Vázquez, P.,Abelairas-Gómez, C., López-García, S., \& Palacios-Aguilar, J. (2017). El Nadador de Rescate SAR. Revisión Histórica. RETOS 31, 145-150. Recuperado de: file:///C:/Users/ M a r \% C 3 \% A D a In m a c u l a d a / D o w n l o a d s / DialnetElNadadorDeRescateSAR-5841362\%20(2).pdf

Vickers, J.N. (2007). Perception, Cognition \& Decision Training: The Quiet Eye in Action. Champaign, IL: Human Kinetics.

Vickers, J.N. (2016). The Quiet eye: Origins, Controversies and Future Directions. Kinesiology Review 5(2), 119-128. https://doi.org/ 10.1123/kr.2016-0005

Williams, A.M., Davids, K., Burwitz, L., \& Williams, J.G. (1994). Visual search strategies in experienced and inexperienced soccer players. Research Quarterly for Exercise and Sport 65(2), 127135.

https://doi.org/10.1080/02701367.1994.10607607

Williams, A. Williams, J.G.P. \& Pascoe, J.G. (1999). Visual perception and action in sport. Londres, Reino Unido: E \& FN SPON

World Health Organization (2008). World report on child injury prevention. Geneva: World Health Organization.

World Health Organization (2011). Informe mundial sobre prevención de las lesiones en los niños.

World Health Organization. (2014). Global Report on Drowning: Preventing a Leading Killer. Geneva, World Health Organization Recuperado de: https://www.who.int/violence_injury_prevention/ global_report_drowning/Final_report_full_web.pdf .

World Health Organization (2016). World report on drowning: Prevent an important cause of mortality. Geneva, World Health Organization.

World Health Organization (2017). Preventing drowning: An implementation guide. Geneva, World Health Organization. Recuperado de: http://apps.who.int/iris/bitstream/handle/10665/ $\begin{array}{lllllll}2 & 5 & 5 & 1 & 9 & 6 & /\end{array}$

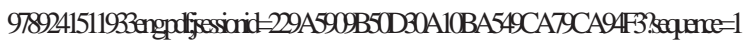

